\title{
Value Regions in Classes of Conformal Mappings
}

\section{V. Prokhorov}

Dmitri V. Prokhorov, https://orcid.org/0000-0002-0490-941X, Saratov State University, 83 Astrakhanskaya St., Saratov 410012, Russia; Petrozavodsk State University, 33 Lenin St., Petrozavodsk 185910, Republic of Karelia, Russia, ProkhorovDV@info.sgu.ru

The survey is devoted to most recent results in the value region problem over different classes of holomorphic univalent functions represented by solutions to the Loewner differential equations both in the radial and chordal versions. It is important also to present classical and modern solution methods and to compare their efficiency. More details are concerned with optimization methods and the Pontryagin maximum principle, in particular. A value region is the set $\left\{f\left(z_{0}\right)\right\}$ of all possible values for the functional $f \mapsto f\left(z_{0}\right)$ where $z_{0}$ is a fixed point either in the upper half-plane for the chordal case or in the unit disk for the radial case, and $f$ runs through a class of conformal mappings. Solutions to the Loewner differential equations form dense subclasses of function families under consideration. The coefficient value regions $\left\{\left(a_{2}, \ldots, a_{n}\right): f(z)=z+\sum_{n=2}^{\infty} a_{n} z^{n}\right\}$, $|z|<1$, are the part of the field closely linked with extremal problems and the Bombieri conjecture about the structure of the coefficient region for the class $S$ in a neighborhood of the point $(2, \ldots, n)$ corresponding to the Koebe function.

Keywords: value region, Loewner equation, reachable set, boundary curve.

Received: 07.04.2018 / Accepted: 12.05.2019 / Published: 31.08.2019

This is an open access article distributed under the terms of Creative Commons Attribution License (CC-BY 4.0).

DOI: https://doi.org/10.18500/1816-9791-2019-19-3-258-279

\section{INTRODUCTION}

Geometric function theory of a complex variable studies metric and geometric properties of conformal mappings $w=f(z)$ from domains in the complex plane $\mathbb{C}$. Due to the seminal Riemann theorem, all simply connected domains with at least two boundary points are conformally equivalent, which means that there is a conformal mapping $f: D \rightarrow B$ from any such domain $D$ onto another domain $B$. Moreover, the function $f$ is unique if it satisfies the normalization conditions $f\left(z_{0}\right)=w_{0}$ and $f^{\prime}\left(z_{0}\right)>0$ at an inner point $z_{0} \in D$ and any $w_{0} \in B$. Therefore, it is convenient to consider Riemann mappings only from (or onto) canonical domains, for example, disks and half-planes.

Extremal problems are the central objects of attention in the geometric function theory of complex variables. The main class under consideration consists of all analytic and univalent functions $f$ in the unit disk $\mathbb{D}=\{z \in \mathbb{C}:|z|<1\}$ normalized by $f(z)=z+\sum_{n=2}^{\infty} a_{n} z^{n}$. This class denoted by $S$ can be thought of as a factor set of all conformal mappings from $\mathbb{D}$ with respect to linear maps $w(z)=a z+b, a \neq 0$. The first results in estimating functionals in the class $S$ and its subclasses demonstrated bounds for $|f(z)|, \arg \{f(z) / z\},\left|f^{\prime}(z)\right|$ depending on $|z|$, coefficients $\left|a_{n}\right|$ for initial values $n$ and others. A wide list of various results is given in the basic monographs [1-4].

Functional estimating is a partial case of the more general and complicated problem to describe a value region for a functional or a system of functionals over different classes of holomorphic mappings. Remind a far-reaching sharpening of the Schwarz lemma due to Rogosinski [5] where the value region $\left\{f\left(z_{0}\right)\right\}$ for a fixed $z_{0} \in \mathbb{D}$ is 
precisely described over the class of holomorphic functions $f(z)$ in $\mathbb{D}, f(0)=0, f^{\prime}(0) \geqslant 0$ and $|f(z)|<1$ for $|z|<1$. The complex-valued functional $L(f)=f\left(z_{0}\right)$ can be regarded as a system of two real-valued functionals $\operatorname{Re} L(f)$ and $\operatorname{Im} L(f)$. Grunsky [6] established the set $\mathscr{W}\left(z_{0}\right):=\left\{\log \left(f\left(z_{0}\right) / z_{0}\right)\right\}, z_{0} \in \mathbb{D}$, within the class $S$.

The most complicated problem concerns with the four-dimensional system of functionals

$$
\left\{\log \left|\frac{f\left(z_{0}\right)}{z_{0}}\right|, \arg \frac{f\left(z_{0}\right)}{z_{0}}, \log \left|f^{\prime}\left(z_{0}\right)\right|, \arg f^{\prime}\left(z_{0}\right)\right\}, \quad 0<\left|z_{0}\right|<1
$$

on the class $S$. Its complete solution was obtained by Popov [7]. Another approach to solve this problem was proposed by Gutlyanskii [8].

Also, mention the impressive description of the real four-dimensional value region

$$
\left\{\left(a_{2}, a_{3}\right): f(z)=z+\sum_{n=2}^{\infty} a_{n} z^{n} \in S\right\}
$$

given by Schaeffer and Spencer in [9].

Among numerous methods to estimate functionals in the class $S$ we point out at the parametric method created mostly by Loewner [10], Kufarev [11] and Pommerenke [12]. In particular, it allows us to represent a dense subclass of $S$ by integrals of the Loewner ordinary differential equation. Gutlyanskii [8] applied the parametric method to solve the value region problem.

Namely, to every function $f$ from a dense subclass of $S$ there corresponds a continuous function $u=u(t), 0 \leqslant t<\infty$, such that $f(z)=\lim _{t \rightarrow \infty} e^{t} w(z, t)$, where $w(z, t)$ is a solution to the Cauchy problem for the Loewner ordinary differential equation

$$
\begin{gathered}
\frac{d w}{d t}=-w \frac{e^{i u(t)}+w}{e^{i u(t)}-w}, \quad w(z, 0)=z, \quad z \in \mathbb{D}, \\
w(z, t)=e^{-t}\left(z+\sum_{n=2}^{\infty} a_{n}(t) z^{n}\right) .
\end{gathered}
$$

Note that if $w(\mathbb{D}, t)=\mathbb{D} \backslash \gamma[0, t]$ with a Jordan curve $\gamma$, then there is a continuous $u$ generating $f$. The converse statement is false, see the Kufarev counterexample in [13].

During last decades, many mathematicians take a growing interest in the class of all conformal self-maps of the upper half-plane $\mathbb{H}=\{z \in \mathbb{C}: \operatorname{Im} z>0\}$. Present the Loewner evolution for $f(\cdot, t): \mathbb{H} \backslash \gamma_{t} \rightarrow \mathbb{H}$.

Consider a growing slit $\gamma[0, t], 0 \leqslant t \leqslant T$, along a Jordan curve. The mapping functions $f(z, t)$, with the hydrodynamic normalization near infinity as

$$
f(z, t)=z+\frac{2 t}{z}+O\left(\frac{1}{|z|^{2}}\right), \quad z \rightarrow \infty,
$$

which map $\mathbb{H} \backslash \gamma[0, t]$ onto $\mathbb{H}$, solve the Loewner ordinary differential equation

$$
\frac{d f(z, t)}{d t}=\frac{2}{f(z, t)-\lambda(t)}, \quad f(z, 0)=z,
$$

with a certain function $\lambda(t)$ that is a real-valued continuous driving term. The number $t$ is called the half-plane capacity of $\gamma[0, t]$. 
Without loss of generality, it is assumed that $\gamma[0, t]$ is emanating from the origin. Then $f^{-}$and $f^{+}$map the left and right sides of $\gamma[0, t]$ onto the two adjoining segments $\left[f^{-}(0, t), \lambda(t)\right]$ and $\left[\lambda(t), f^{+}(0, t)\right]$ in $\mathbb{R}=\partial \mathbb{H}$, respectively,

$$
\lambda(0)=0, \quad f^{-}(0,0)=f^{+}(0,0)=0 .
$$

To distinguish the two versions of the Loewner evolution, and following modern denotations, call (1) the radial differential equation while (2) is the chordal differential equation.

This survey is aimed to present recent results on value regions over classes of conformal mappings from $\mathbb{D}$ or $\mathbb{H}$. Almost all of them are based on the parametric method and especially on the optimality approach and the Pontryagin maximum principle developed in the frames of the Loewner theory. In this setting, a value region is identified as a reachable set for a system of ordinary differential equations generated by Loewner equations (1) or (2).

\section{REACHABLE SETS FOR THE CHORDAL LOEWNER EQUATION}

We are focused at the problem to find value ranges for the complex-valued functional $\left\{f\left(z_{0}\right)\right\}, z_{0} \in \mathbb{H}$, in classes of conformal mappings from $\mathbb{H}$ or subsets of $\mathbb{H}$. Along with Gogosinski's [5] and Grunsky's [6] similar results for $z_{0} \in \mathbb{D}$, add the extension by Goryainov and Gutlyanskii [14] who gave a description of the same set $\left\{f\left(z_{0}\right)\right\}, z_{0} \in \mathbb{D}$, over the subclass $S(M)$ of bounded functions $f \in S,|f(z)|<M$ in $\mathbb{D}, M>1$.

An analogue of Rogosinski's result for univalent functions was obtained by Roth and Schleissinger [15] in terms of hyperbolic geometry. They gave an analogous description of the value regions $\left\{f\left(z_{0}\right)\right\}, z_{0} \in \mathbb{D}$, over the class of univalent holomorphic functions $f: \mathbb{D} \rightarrow \mathbb{D}, f(0)=0, f^{\prime}(0)>0$, and $\left\{g\left(z_{0}\right)\right\}, z_{0} \in \mathbb{H}$, over the class of functions $g: \mathbb{H} \rightarrow \mathbb{H}$ with the hydrodynamic normalization. Their proofs are based on the radial and chordal Loewner equations. In particular, they proved the following theorem for the reachable set

$$
\mathscr{R}\left(z_{0}\right)=\left\{g\left(z_{0}, t\right), t \geqslant 0\right\}, \quad z_{0} \in \mathbb{H},
$$

over the class of functions $g(z, t)$ having the hydrodynamic normalization at infinity and such that $g(z, \cdot)$ solve equation

$$
\frac{d g(z, t)}{d t}=\frac{-2}{g(z, t)-\lambda(t)}, \quad g(z, 0)=z,
$$

with some continuous driving function $\lambda:[0, \infty) \rightarrow \mathbb{R}$.

Theorem 1 ([15]). Let $z_{0} \in \mathbb{H}$. Then

$$
\mathscr{R}\left(z_{0}\right)=\left\{z \in \mathbb{H}: \operatorname{Im} z>\operatorname{Im} z_{0}\right\} \cup\left\{z_{0}\right\} .
$$

We will extend these results to functions with a fixed time $T$. Let $K \subset \mathbb{H}$ be bounded and $\bar{K}$ be the closure of $K$. The set $K$ is a hull if $K=\mathbb{H} \cap \bar{K}$ and $\mathbb{H} \backslash K$ are connected and simply connected. Denote $\mathscr{H}(T)$ the set of conformal maps from $\mathbb{H} \backslash K(T)$ having the hydrodynamic normalization, with arbitrary hulls $K=K(T)$ of half-plane capacity $T$, onto $\mathbb{H}$. The problem is to find a value region

$$
\left\{f\left(z_{0}\right): f \in \mathscr{H}(T), z_{0} \notin K(T)\right\}, \quad z_{0} \in \mathbb{H} .
$$


As far as $\mathscr{H}(T)$ possesses the translation and scaling properties, it is possible to reduce the value region problem to the case $z_{0}=i$. So let us describe the value region

$$
D(T):=\{f(i): f \in \mathscr{H}(T), i \notin K(T)\} .
$$

Interpret the result by Roth and Schleissinger as a description of the complementary set $\mathbb{H} \backslash \cup_{T>0} D(T)$.

For $0 \leqslant T \leqslant \frac{1}{4}$, denote by $C=C_{0}(\varphi, T)>0,-\frac{\pi}{2}<\varphi<\frac{\pi}{2}$, the unique root of the equation

$$
2 \cos ^{2} \varphi \log (1-\sin \varphi)+(1-\sin \varphi)^{2}=2 \cos ^{2} \varphi \log C+C^{2}(1-4 T) .
$$

The following theorems $2-4$ are proved in [16].

Theorem 2. The domain $D(T), 0<T \leqslant \frac{1}{4}$, is bounded by two curves $l_{1}$ and $l_{2}$ connecting the points $i$ and $i \sqrt{1-4 T}$. The curve $l_{1}$ in the complex $(u, v)$-plane is parameterized by the equations

$$
\begin{gathered}
u(T)=\frac{C_{0}^{2}(\varphi, T)(4 T-1)+(1-\sin \varphi)^{2}}{2 C_{0}(\varphi, T) \cos \varphi}, v(T)=\frac{1-\sin \varphi}{C_{0}(\varphi, T)}, \\
-\frac{\pi}{2}<\varphi<\frac{\pi}{2} .
\end{gathered}
$$

The curve $l_{2}$ is symmetric to $l_{1}$ with respect to the imaginary axis. Every point $w=u+i v \in \partial D(T) \backslash\{i\}$ corresponds to a unique function from $\mathscr{H}(T)$.

Generalize the problem posed in Theorem 2 and consider a value region $D(T)$ with $T>\frac{1}{4}$ with possible singular solutions to the chordal Loewner equation, i.e., the point $w=i$ may belong to $K(T)$. In this case, the closure of $D(T)$ contains a segment $I(T) \subset \mathbb{R}$. Among boundary functions corresponding to a point $w \in I(T)$ there is a function $f_{K(T)}$ which admits a continuous extension on the union of $\mathbb{H} \backslash K(T)$ and the both sides of a curvilinear slit $K(T)$, so that the extended function $f_{K(T)}$ maps $z=i$ in $I(T)$. We will see in the next theorem that $I(T)$ coincides with the whole axis $\mathbb{R}$. Preserve the denotation $D(T)$ for value regions over this generalized class of functions.

Introduce new denotations. Denote by $\varphi_{0}(T) \in\left(-\frac{\pi}{2}, \frac{\pi}{2}\right), T>\frac{1}{4}$, the unique solution of the equation

$$
\log \frac{1-\sin \varphi}{1+\sin \varphi}+\frac{1-\sin \varphi}{1+\sin \varphi}+1=\log \frac{1}{4 T-1} .
$$

For $T>\frac{1}{4}$, this equation has a unique solution $\varphi=\varphi_{0}(T)$ depending on $T$. For $T>\frac{1}{4}$ and $\varphi \in\left[\varphi_{0}(T), \frac{\pi}{2}\right]$, denote by $C_{0}(\varphi, T)>0$ the minimal root of equation (4) and by $C_{00}(\varphi, T)>0$ the maximal root of equation (4).

Note that, for $T>\frac{1}{4}$ and $\varphi \in\left(\varphi_{0}(T), \frac{\pi}{2}\right)$, equation (4) has exactly two roots.

Theorem 3. The domain $D(T), T>\frac{1}{4}$, is bounded by two curves $l_{1}=l_{11} \cup l_{12}$ and $l_{2}=l_{21} \cup l_{22}$ having a mutual point $i \in l_{11} \cap l_{21}$. The curve $l_{11}$ in the complex $(u, v)$-plane, for $\varphi \in\left[\varphi_{0}(T), \frac{\pi}{2}\right]$, is parameterized by the equations

$$
u(T)=\frac{C_{0}^{2}(\varphi, T)(4 T-1)+(1-\sin \varphi)^{2}}{2 C_{0}(\varphi, T) \cos \varphi}, \quad v(T)=\frac{1-\sin \varphi}{C_{0}(\varphi, T)} .
$$


The curve $l_{12}$, for $\varphi \in\left[\varphi_{0}(T), \frac{\pi}{2}\right]$, is parameterized by the equation

$$
u(T)=\frac{C_{00}^{2}(\varphi, T)(4 T-1)+(1-\sin \varphi)^{2}}{2 C_{00}(\varphi, T) \cos \varphi}, \quad v(T)=\frac{1-\sin \varphi}{C_{00}(\varphi, T)} .
$$

The curve $l_{2}$ is symmetric to $l_{1}$ with respect to the imaginary axis.

Following Roth and Schleissinger, develop the results towards inverse functions. Denote by $\mathscr{H}^{*}(T), T>0$, the class of functions $g(w)=f^{-1}(w)$ for $f \in \mathscr{H}(T)$. Denote

$$
D^{*}(T)=\left\{g(i): g \in \mathscr{H}^{*}(T)\right\}, \quad T>0 .
$$

It follows from the results by Roth and Schleissinger that

$$
\cup_{T>0} D^{*}(T)=\{w \in \mathbb{C}: \operatorname{Im} w>1\} \cup\{i\} .
$$

Denote by $C^{0}(\varphi, T)>0,-\frac{\pi}{2}<\varphi<\frac{\pi}{2}, T \geqslant 0$, a unique root of the equation

$$
2 \cos ^{2} \varphi \log (1+\sin \varphi)+(1+\sin \varphi)^{2}=2 \cos ^{2} \varphi \log C+C^{2}(1+4 T) .
$$

Theorem 4. The domain $D^{*}(T), T>0$, is bounded by two curves $L_{1}$ and $L_{2}$ connecting the points $i$ and $i \sqrt{1+4 T}$. The curve $L_{1}$ in the complex $(u, v)$-plane is parameterized by the equations

$$
\begin{gathered}
u(T)=\frac{\left(C^{0}(\varphi, T)\right)^{2}(4 T+1)-(1+\sin \varphi)^{2}}{2 C^{0}(\varphi, T) \cos \varphi}, v(T)=\frac{1+\sin \varphi}{C^{0}(\varphi, T)}, \\
-\frac{\pi}{2}<\varphi<\frac{\pi}{2} .
\end{gathered}
$$

The curve $L_{2}$ is symmetric to $L_{1}$ with respect to the imaginary axis.

Remark that Zherdev [17] developed the results and methods in [16] and described the value region for solutions to the chordal Loewner ODE (2) with $T \leqslant \frac{1}{4}$ under the restriction $|\lambda(t)| \leqslant c, c \geqslant 0$, for the driving function $\lambda$ in (2). Besides, he managed to write down explicitly the parametric representation of the boundary of the domain $D(T)$ in the $W$-plane, $W=X+i Y$, as follows:

$$
2 X^{2}=\left(1-4 T-Y^{2}\right) \log Y, \quad \sqrt{1-4 T} \leqslant Y \leqslant 1 .
$$

It is important to mention that the value region $\left\{f\left(z_{0}\right)\right\}$ in $\mathscr{H}(T)$ is the reachable set of the chordal Loewner ODE, i.e., this is a set of values $f\left(z_{0}, T\right)$ for all possible trajectories $f\left(z_{0}, t\right)$ of the Loewner ODE emanating from $f\left(z_{0}, 0\right)=z_{0}$.

Recently, several other authors widely applied optimization methods to describe value regions in subclasses of univalent functions. In particular, indicate the Ph.D. thesis of Koch [18] submitted in 2016. Mainly her results relate to mappings from the unit disk $\mathbb{D}$. In this section, we will be acquainted with two Koch's results for symmetric maps in the upper half-plane $\mathbb{H}$.

In analogy to typically real functions in the radial setting, Koch considered functions which possess certain symmetric properties. Namely, let

$$
\mathscr{I}=\left\{f: f^{-1} \in \cup_{T>0} \mathscr{H}^{*}(T), f(-\bar{z})=-\overline{f(z)}, z \in \mathbb{H}\right\} .
$$

Define

$$
V_{\mathscr{I}}\left(z_{0}\right)=\left\{f\left(z_{0}\right): f \in \mathscr{I}\right\} .
$$

The two following theorems belong to Koch [18]. 
Theorem 5. Let $z_{0} \in \mathbb{H}$. If $\operatorname{Re} z_{0}=0$, then

$$
V_{\mathscr{I}}\left(z_{0}\right)=\left\{z_{0}+i t: t \geqslant 0\right\} .
$$

Next, assume that $\operatorname{Re} z_{0}>0$ and define the two curves $C\left(z_{0}\right)$ and $D\left(z_{0}\right)$ by

$$
\begin{gathered}
C\left(z_{0}\right)=\left\{\sqrt{z_{0}^{2}-4 t}: t \geqslant 0\right\}=\left\{x+i y \in \mathbb{H}: x y=\operatorname{Re} z_{0}, \operatorname{Im} z_{0}, x \in\left(0, \operatorname{Re} z_{0}\right]\right\}, \\
D\left(z_{0}\right)=\left\{z_{0}+e^{i \arg z_{0}} t: t \geqslant 0\right\} .
\end{gathered}
$$

Then the closure $\overline{V_{\mathscr{I}}\left(z_{0}\right)}$ of the set $V_{\mathscr{I}}\left(z_{0}\right)$ is the closed subset of $\mathbb{H}$ bounded by $C\left(z_{0}\right)$ and $D\left(z_{0}\right)$, and

$$
V_{\mathscr{I}}\left(z_{0}\right)=\left\{z_{0}\right\} \cup \overline{V_{\mathscr{I}}\left(z_{0}\right)} \backslash D\left(z_{0}\right) .
$$

The case $\operatorname{Re} z_{0}<0$ follows from the case $\operatorname{Re} z_{0}>0$ by reflection with respect to the imaginary axis.

The value set $\left\{f^{-1}\left(z_{0}\right)\right\}$ for the inverse functions is given similarly.

Theorem 6. Let $z_{0} \in \mathbb{H}$. Define

$$
V_{\mathscr{I}}^{*}\left(z_{0}\right)=\left\{f^{-1}\left(z_{0}\right): f \in \mathscr{I}, z_{0} \in f(\mathbb{H})\right\} .
$$

If $\operatorname{Re} z_{0}=0$, then

$$
V_{\mathscr{I}}^{*}\left(z_{0}\right)=\left\{z_{0}-i t, t \in\left[0, \operatorname{Im} z_{0}\right]\right\} .
$$

Next, assume that $\operatorname{Re} z_{0}>0$ and define the two curves $C^{*}\left(z_{0}\right)$ and $D^{*}\left(z_{0}\right)$ by

$$
\begin{gathered}
C^{*}\left(z_{0}\right)=\left\{\sqrt{z_{0}^{2}+4 t}: t \geqslant 0\right\}=\left\{x+i y \in \mathbb{H}: x y=\operatorname{Re} z_{0} \operatorname{Im} z_{0}, x \geqslant \operatorname{Re} z_{0}\right\}, \\
D^{*}\left(z_{0}\right)=\left\{z_{0}-e^{i \arg z_{0}} t: t \in\left[0,\left|z_{0}\right|\right]\right\} .
\end{gathered}
$$

Then the closure $\overline{V_{\mathscr{I}}^{*}\left(z_{0}\right)}$ of the set $V_{\mathscr{I}}^{*}\left(z_{0}\right)$ is the closed subset of $\mathbb{H}$ bounded by the curves $C^{*}\left(z_{0}\right), D^{*}\left(z_{0}\right)$ and the positive real half-axis. The set $V_{\mathscr{I}}^{*}\left(z_{0}\right)$ is given by

$$
V_{\mathscr{I}}^{*}\left(z_{0}\right)=\left\{z_{0}\right\} \cup \overline{V_{\mathscr{I}}^{*}\left(z_{0}\right)} \backslash\left(D^{*}\left(z_{0}\right) \cup[0, \infty)\right) .
$$

The case $\operatorname{Re} z_{0}<0$ is symmetric.

\section{REACHABLE SETS AND OPTIMIZATION METHODS}

Optimization methods applied in the previous Section are rather effective in the reachable set problem for controllable systems of ordinary differential equations, such as the Loewner ODE. A reachable set of a system of ODE is a set of endpoints of all its trajectories starting from a given point and spread during a fixed time $T$. In other words, this is the set of points which can be reached by solutions to the system of ODE from the initial point up to the moment $T$. Mainly, the value region problems over function classes represented by the Loewner ODE are reduced to the reachable set problems for systems of ODE generated by the Loewner ODE.

To solve the problem, we consider a set of target functionals corresponding to the boundary points of the reachable set and construct a Hamiltonian system with a driving function satisfying the Pontryagin maximum principle. This allows us to obtain a set of 
boundary problems for phase and adjoint systems of ODE and reformulate it for a set of the Cauchy problems for these systems.

Shortly, this is a universal optimization scheme giving necessary local optimum criteria. It remains to show that, in concrete cases, these criteria are global and sufficient.

Usually, we arrive at a final result when a set of all boundary points satisfying the necessary optimum conditions is a closed Jordan curve (or a closed simple surface in higher dimensions).

First, the optimization ideas in geometric function theory have been announced by Goodman, the last Loewner's Ph.D. student, at the International Congress of Mathematicians, 1966, in Moscow. Later, Goodman exposed the approach in his Ph.D. thesis [19] where he combined Loewner's theory with the then new Pontryagin maximum principle. More details appeared in papers by Friedland and Schiffer [20,21].

Saratov mathematicians applied the optimization method to solve a lot of extremal problems in different classes of univalent functions represented by the Loewner parametrization. Their contribution has been mentioned, e.g., in Roth's Ph.D. thesis [22], which is devoted to reachable sets of certain control systems in the class $\mathscr{H}(\mathbb{D})$ that is the set of all analytic functions in $\mathbb{D}$.

In particular, Roth wrote [22, p. i-ii]: “...we consider coefficient problems for univalent functions as optimal control problems for certain finite-dimensional control systems. This point of view was initiated and successfully employed by G. S. Goodman, S. Friedland and M. M. Schiffer and more recently by D. V. Prokhorov in a series of papers". And more [22, p. 6]: "Whenever a class of analytic functions is representable as a reachable set, Pontryagin's maximum principle applies and gives immediately some necessary conditions for the solutions of extremal problems in this class". In his thesis, Roth turned to the formulation of the so-called principle of optimality for control systems in $\mathscr{H}(\mathbb{D})$. He discussed two control systems in $\mathbb{C}^{n}$ due to Friedland and Schiffer [21] and Prokhorov $[23,24]$, respectively, whose reachable sets coincide with the so-called coefficient region of functions univalent in $\mathbb{D}$. Roth concludes that the two methods are essentially equivalent and compares their interrelation with Schiffer's differential equation [25].

Give a sketch of the Hamiltonian formalism. A dynamical system in $\mathbb{C}^{2 n}$ is Hamiltonian if it is of the form

$$
\dot{x}=\nabla_{s} H(x),
$$

where $\nabla_{s}$ denotes the symplectic gradient given by

$$
\nabla_{s}=\left(\frac{\partial}{\partial \bar{x}_{n+1}}, \ldots, \frac{\partial}{\partial \bar{x}_{2 n}},-\frac{\partial}{\partial x_{1}}, \ldots,-\frac{\partial}{\partial x_{n}}\right) .
$$

The function $H$ is the Hamiltonian function of the system. It is convenient to redefine the coordinates $\left(x_{n+1}, \ldots, x_{2 n}\right)=\left(\psi_{1}, \ldots, \psi_{n}\right)$ and rewrite the system as

$$
\dot{x}_{k}=\frac{\partial H}{\partial \overline{\psi_{k}}}, \quad \dot{\psi_{k}}=-\frac{\partial H}{\partial x_{k}}, \quad k=1,2, \ldots n .
$$

The two-form $\omega=\sum_{k=1}^{n} d x \wedge d \bar{\psi}$ admits the Poisson bracket $\{\cdot, \cdot\}$,

$$
\{f, g\}=\sum_{k=1}^{n}\left(\frac{\partial f}{\partial x_{k}} \frac{\partial g}{\partial \overline{\psi_{k}}}-\frac{\partial f}{\partial \overline{\psi_{k}}} \frac{\partial g}{\partial x_{k}}\right)
$$

associated with $\omega$. 
The first integrals $\Phi$ of the system are characterized by $\{\Phi, H\}=0$. If the Hamiltonian system admits $1 \leqslant k<n$ independent involutory integrals, then it is called partially integrable in the sense of Liouville.

In [26], for a system generated by the Loewner equation, the authors evaluated explicitly the first integrals $\left(\Phi_{1}, \ldots, \Phi_{n}\right)$ so that $\left[\frac{n}{2}\right]$ first integrals $\left(\Phi_{[n / 2]+1}, \ldots, \Phi_{n}\right)$ are pairwise involutory and the integrals $\left(\Phi_{1}, \ldots, \Phi_{[n / 2]}\right)$ are not pairwise involutory but their Poisson brackets give all the rest of the integrals.

Roth [27] revisited the control-theoretic interpretation of the Loewner equation. He writes that [26, p. 94]: “...this approach has been used by many others, see e.g. $[2,20,21,28]$ and in particular the important contributions by Prokhorov [23,24,29,30]". Also [26, p. 96]: "The idea of viewing the value region as reachable sets of the Loewner equation has been pioneered by Prokhorov $[23,24,30]$ ". Roth poses the problem to prove Teichmüller's coefficient theorem using only the Loewner differential equation. He notes that the standard method in control theory for obtaining sufficient conditions for optimal functions makes use of Bellman functions and refers to [24] for some applications of Bellman functions to the Loewner equation.

\section{REACHABLE SETS FOR THE RADIAL LOEWNER EQUATION}

Going back to results by Roth and Schleissinger [15] introduce the class of univalent self-maps $f: \mathbb{D} \rightarrow \mathbb{D}$. Let

$$
\mathscr{S}_{T}=\left\{f: \mathbb{D} \rightarrow \mathbb{D} \text { univalent, } f^{\prime}(0)=e^{-T}\right\}, \quad T>0 .
$$

Note that if $f \in \mathscr{S}_{T}$ then $e^{T} f$ belongs to the class $S(M)$ of bounded functions from $S$ with $M=e^{T}$. For $z_{0} \in \mathbb{D} \backslash\{0\}$, denote

$$
V_{T}\left(z_{0}\right)=\left\{f\left(z_{0}\right): f \in \mathscr{S}_{T}\right\} .
$$

Roth and Schleissinger [15] described the set $\cup_{T>0} V_{T}\left(z_{0}\right)$. To formulate their result, endow $\mathbb{D}$ with the standard hyperbolic metric

$$
\lambda_{\mathbb{D}}(z) d z=\frac{2|d z|}{1-|z|^{2}} .
$$

The induced hyperbolic distance $d_{\mathbb{D}}(z, w)$ between $z \in \mathbb{D}$ and $w \in \mathbb{D}$ is then given by

$$
d_{\mathbb{D}}(z, w)=\log \frac{1+|(z-w) /(1-\bar{w} z)|}{1-|(z-w) /(1-\bar{w} z)|} .
$$

Theorem 7 ([15]). Let $z_{0} \in \mathbb{D} \backslash\{0\}$. Then

$$
\cup_{T>0} V_{T}\left(z_{0}\right) \cup\{0\}=\left\{z=|z| e^{i \varphi} \in \mathbb{D}: d_{\mathbb{D}}(0, z)-d_{\mathbb{D}}\left(0, z_{0}\right) \leqslant-\left|\varphi-\arg z_{0}\right|, \varphi \in \mathbb{R}\right\} .
$$

This theorem is known as Grunsky's result [6]. For fixed $T$, it was proved earlier by Goryainov and Gutlyanski [14] in another setting. Roth and Schleissinger comment their theorem as [15, p. 1102]: "The results in [6,14] are much more difficult to prove. The purpose is to give a simple and direct proof".

The three following theorems belong to Koch and Schleissinger [31]. Due to rotations in $\mathscr{S}_{T}$, a choice of $z_{0} \in \mathbb{D} \backslash\{0\}$ in the value region problem for $V_{T}\left(z_{0}\right)$ is reduced to $z_{0} \in(0,1)$. 
Theorem 8. Let $z_{0} \in(0,1)$. For $x_{0} \in[-1,1]$ and $T>0$, let $r=r\left(T, x_{0}\right)$ be the unique solution to the equation

$$
\begin{gathered}
\left(1+x_{0}\right)\left(1-z_{0}\right)^{2} \log (1-r)+\left(1-x_{0}\right)\left(1+z_{0}\right)^{2} \log (1+r)- \\
\left(1-2 x_{0} z_{0}+z_{0}^{2}\right) \log r=\left(1+x_{0}\right)\left(1-z_{0}\right)^{2} \log \left(1-z_{0}\right)+ \\
\left(1-x_{0}\right)\left(1+z_{0}\right)^{2} \log \left(1+z_{0}\right)-\left(1-2 x_{0} z_{0}+z_{0}^{2}\right) \log e^{-T} z_{0}
\end{gathered}
$$

and let

$$
\sigma\left(T, x_{0}\right)=\frac{2\left(1-z_{0}\right)^{2} \sqrt{1-x_{0}^{2}}}{1-2 x_{0} z_{0}+z_{0}^{2}}\left(\operatorname{arctanh} z_{0}-\operatorname{arctanh} r\left(T, x_{0}\right)\right) .
$$

Furthermore, for fixed $T \geqslant 0$, define the two curves $C_{+}\left(z_{0}\right)$ and $C_{-}\left(z_{0}\right)$ by

$$
C_{ \pm}\left(z_{0}\right)=\left\{w_{ \pm}\left(x_{0}\right):=r\left(T, x_{0}\right) e^{ \pm \sigma\left(T, x_{0}\right)}: x_{0} \in[-1,1]\right\} .
$$

Then, if $\operatorname{arctanh} z_{0}<\frac{\pi}{2}, V_{T}\left(z_{0}\right)$ is the closed region whose boundary consists of the two curves $C_{ \pm}\left(z_{0}\right)$, which intersect at $x_{0}$. For $\operatorname{arctanh} z_{0} \geqslant \frac{\pi}{2}$, there are two different cases. First assume that $T$ is large enough that the equation $\sigma(t, x)=\pi$ admits a solution $x \in[-1,1]$. Then the curves $C_{ \pm}\left(z_{0}\right)$ intersect more than twice. There is a $\chi \in(-1,1)$ such that $\tilde{C}_{+}\left(z_{0}\right) \cup \tilde{C}_{-}\left(z_{0}\right)$ is a closed Jordan curve, where $\tilde{C}_{ \pm}\left(z_{0}\right)=\left\{w_{ \pm}\left(x_{0}\right): x_{0} \in[\chi, 1]\right\}$, and an $\mathscr{N} \in(-1,1)$ such that $\hat{C}_{+}\left(z_{0}\right) \cup \hat{C}_{-}\left(z_{0}\right)$ is a closed Jordan curve, where $\hat{C}_{ \pm}\left(z_{0}\right)=\left\{w_{ \pm}\left(x_{0}\right): x_{0} \in[-1, \mathscr{N}]\right\}$. Then $V_{T}\left(z_{0}\right)$ is the closed region whose boundary is the union of $\tilde{C}_{ \pm}\left(z_{0}\right)$ and $\hat{C}_{ \pm}\left(z_{0}\right)$. For smaller $T, V_{T}\left(z_{0}\right)$ can be described as in the first case.

Again, we refer to the result due to Goryainov and Gutlyanski [14] in another setting. Koch and Schleissinger explain the proof of their theorem as [31, p. 1773]: "We use a different and more straightforward approach to directly determine the set $V_{T}\left(z_{0}\right)$ by applying Pontryagin's maximum principle to the radial Loewner equation”.

Let

$$
\mathscr{W}\left(z_{0}\right)=\left\{f^{-1}\left(z_{0}\right): f \in \cup_{T>0} \mathscr{S}_{T} \text { with } z_{0} \in f(\mathbb{D}\} .\right.
$$

Theorem 9. We have

$$
\mathscr{W}\left(z_{0}\right)=\left\{r e^{i \sigma}: d_{\mathbb{D}}(0, r) \geqslant|\sigma|+d_{\mathbb{D}}\left(0, z_{0}\right), \sigma \in[-\pi, \pi]\right\} .
$$

Furthermore, determine the value set

$$
\mathscr{W}_{T}\left(z_{0}\right)=\left\{f^{-1}\left(z_{0}\right): f \in \mathscr{S}_{T} \text { with } z_{0} \in f(\mathbb{D})\right\}
$$

and $\overline{W_{T}\left(z_{0}\right)}$ is the closure of $W_{T}\left(z_{0}\right)$. Evidently, $\mathscr{W}\left(z_{0}\right)=\cup_{T>0} \mathscr{W}_{T}\left(z_{0}\right)$.

Theorem 10. Let $z_{0} \in(0,1)$. For $x_{0} \in[-1,1]$ and $T>0$, let $r=r\left(T, x_{0}\right)$ be the unique positive solution to the equation

$$
\begin{aligned}
& \left(1-x_{0}\right)\left(1-z_{0}\right)^{2} \log (1-r)+\left(1+x_{0}\right)\left(1+z_{0}\right)^{2} \log (1+r)- \\
& -\left(1+2 x_{0} z_{0}+z_{0}^{2}\right) \log r=\left(1-x_{0}\right)\left(1-z_{0}\right)^{2} \log \left(1-z_{0}\right)+ \\
& +\left(1+x_{0}\right)\left(1+z_{0}\right)^{2} \log \left(1+z_{0}\right)-\left(1+2 x_{0} z_{0}+z_{0}^{2}\right) \log e^{T} z_{0}
\end{aligned}
$$


and let

$$
\sigma\left(T, x_{0}\right)=\frac{2\left(1-z_{0}\right)^{2} \sqrt{1-x_{0}^{2}}}{1+2 x_{0} z_{0}+z_{0}^{2}}\left(\operatorname{arctanh} r\left(t, X_{0}\right)-\operatorname{arctanh} z_{0}\right) .
$$

If

$$
T<T^{*}:=\log \frac{\left(1+z_{0}\right)^{2}}{4 z_{0}},
$$

then $r\left(T, x_{0}\right)$ can be extended continuously to $x_{0}=1, \mathscr{W}_{T}\left(z_{0}\right)=\overline{\mathscr{W}_{T}\left(z_{0}\right)}$, and $\mathscr{W}_{T}\left(z_{0}\right)$ is the closed region bounded by the two curves $D_{ \pm}\left(z_{0}\right)=\left\{r\left(T, x_{0}\right) e^{ \pm i \sigma\left(T, x_{0}\right)}: x_{0} \in[-1,1]\right\}$. For $T \geqslant T^{*}$, define the two curves $\tilde{D}_{ \pm}\left(z_{0}\right)=\left\{r\left(T, x_{0}\right) e^{ \pm i \sigma\left(T, x_{0}\right)}: x_{0} \in[-1,1]\right\}$. We have two cases: if $T$ is small enough that $\tilde{D}_{ \pm}\left(z_{0}\right)$ intersect only at $x_{0}=-1$, then $\overline{\mathscr{W}_{T}\left(z_{0}\right)}$ intersects $\partial \mathbb{D}$ and is bounded by two curves $\tilde{D}_{ \pm}\left(z_{0}\right)$ and by the part of $\partial \mathbb{D}$ between the intersection points with the curves which includes the point 1. Otherwise, the two curves intersect on $(-1,1)$ for the first time for some $x_{0}=\chi \in(-1,1)$ and $\overline{\mathscr{W}}_{T}\left(z_{0}\right)$ is the closed region bounded by $\partial \mathbb{D}$ and the two curves $\hat{D}_{ \pm}\left(z_{0}\right)=\left\{r\left(T, x_{0}\right) e^{ \pm i \sigma\left(T, x_{0}\right)}: x_{0} \in[-1, \chi]\right\}$. In the last two cases $\mathscr{W}_{T}\left(z_{0}\right)=\overline{\mathscr{W}_{T}\left(z_{0}\right)} \cap \mathbb{D}$.

The two following theorems on value regions belong to Koch and Schleissinger [32]. Denote by $\mathscr{T}$ the class of analytic typically real self-mappings of $\mathbb{D}$ normalized as $f(0)=0$ and $f^{\prime}(0)>0$. For $\tau \in(0,1]$, denote similarly $\mathscr{T}(\tau)$ the class of functions $f \in \mathscr{T}$ with the restriction $f^{\prime}(0)=\tau$. Remind that typically real functions $f$ in $\mathbb{D}$ are characterized by the condition $\operatorname{Im} z \operatorname{Im} f(z) \geqslant 0$ in $\mathbb{D}$. Let

$$
V_{\mathscr{T}(\tau)}\left(z_{0}\right)=\left\{f\left(z_{0}\right): f \in \mathscr{T}(\tau)\right\} .
$$

Theorem 11. Let $z_{0} \in \mathbb{D} \backslash\{0\}$ and $\tau \in(0,1]$. The set $V_{\mathscr{T}(\tau)}\left(z_{0}\right)$ is the image of the closed region bounded by the two circular arc

$$
\left\{1+\frac{4 \tau z_{0}}{1-2 y z_{0}+z_{0}^{2}}: y \in[2 \tau-1,1]\right\}
$$

and

$$
\left\{\frac{\left(z_{0}+1\right)^{2}\left(1+z_{0}\left(-4+4 \tau-2 x+z_{0}\right)\right)}{\left(z_{0}-1\right)^{2}\left(1-2 x z_{0}+z_{0}^{2}\right)}: x \in[-1,2 \tau-1]\right\}
$$

under the map $w \mapsto \frac{\sqrt{w}-1}{\sqrt{w}+1}$.

Note that the value regions $V_{\mathscr{T}(\tau)}\left(z_{0}\right)$ for all analytic functions and for univalent functions with real Taylor coefficients coincide.

Denote by $\mathscr{R} \geqslant$ the class of all analytic self-mappings $f$ of $\mathbb{D}$ normalized as $f(0)=0$, $f^{\prime}(0) \geqslant 0$, and having only real Taylor coefficients around the origin. Let

$$
V_{\mathscr{R}} \geqslant\left(z_{0}\right)=\left\{f\left(z_{0}\right): f \in \mathscr{R}^{\geqslant}\right\} .
$$

Theorem 12. Let $z_{0} \in \mathbb{D} \backslash\{0\}$. Then $V_{\mathscr{R} \geqslant}\left(z_{0}\right)$ is the closed convex region bounded by the following three curves:

$$
\begin{gathered}
A=\left\{\frac{z_{0}\left(z_{0}-x\right)}{z_{0} x-1}: x \in[0,1]\right\}, \quad B=\left\{\frac{z_{0}\left(z_{0}+x\right)}{z_{0} x+1}: x \in[0,1]\right\}, \\
C=\left\{\frac{z_{0}^{2}\left(z_{0}+2 x-1\right)}{1+2 x z_{0}-z_{0}}: x \in[0,1]\right\} .
\end{gathered}
$$




\section{REACHABLE SETS FOR UNIVALENT FUNCTIONS WITH REAL TAYLOR COEFFICIENTS}

Among other works in the value region problem, select Fedorov's paper [33] concerning with the set $\left\{f\left(z_{0}\right)\right\}, z_{0} \in \mathbb{D}$, over the class $S_{R} \subset S$ of functions $f \in S$ with real Taylor coefficients $a_{n}, n \geqslant 1$, around 0 .

For real $z_{0}$, the problem is trivial since $\left\{f\left(z_{0}\right)\right\}$ is described by one of the following sharp inequalities,

$$
\frac{-\left|z_{0}\right|}{\left(1-\left|z_{0}\right|\right)^{2}} \leqslant f\left(-\left|z_{0}\right|\right) \leqslant \frac{-\left|z_{0}\right|}{\left(1+\left|z_{0}\right|\right)^{2}}, \quad \frac{\left|z_{0}\right|}{\left(1+\left|z_{0}\right|\right)^{2}} \leqslant f\left(\left|z_{0}\right|\right) \leqslant \frac{\left|z_{0}\right|}{\left(1-\left|z_{0}\right|\right)^{2}} .
$$

On the contrary, the problem appeared to be far from triviality in the case of non-real $z_{0}$. Fedorov completely solved the problem by simultaneously considering two moduli problems for pairs of homotopic classes of curves. Partial results in this problem were obtained earlier by Jenkins [34].

Define the class of bounded univalent functions

$$
S_{R}(M)=\left\{f \in S_{R}:|f(z)|<M, z \in \mathbb{D}\right\}, \quad M>1,
$$

with real Taylor coefficients around 0. Usually, extremum problems in subclasses of bounded functions are more difficult than in the whole classes. In the parametric method, theoretically, solutions of extremum problems for bounded functions are of comparative difficulty with unbounded maps. The main difference between the two versions is in constructing an optimal behavior of driving functions either on the maximal set $[0, \infty)$ of time-parameter or on its subset $[0, \log M]$.

Reduce the value region problem to the optimal control problem in the frames of the Loewner theory. Every function $f$ corresponding to a boundary point of the value region $\left\{f\left(z_{0}\right)\right\}$ over $S_{R}(M)$ is given by

$$
f(z)=M w(z, \log M)
$$

where $w(z, t)=e^{-t}\left(z+a_{2}(t) z^{2}+\ldots\right)$ solves the Loewner differential equation

$$
\frac{d w}{d t}=-w \frac{1-w^{2}}{1-u w+w^{2}}, \quad t \geqslant 0, \quad w(z, 0)=z, \quad z \in \mathbb{D},
$$

with a piecewise continuous driving term $u=u(t),-2 \leqslant u \leqslant 2$, having at most one discontinuity point. The set $\left\{f\left(z_{0}\right)\right\}$ in $S_{R}(M)$ is a reachable set for this Loewner ODE at $t=\log M$ with the initial point $z_{0}$.

In [35], the authors described the value region $\left\{f\left(z_{0}\right)\right\}$ in $S_{R}(M)$ in an extremely implicit form. They proved that the set $\left\{f\left(z_{0}\right)\right\}$ in $S_{R}(M)$ is bounded by the two simple curves $\gamma_{1}$ and $\gamma_{2}$. The boundary curve $\gamma_{1}$ is given by

$$
\gamma_{1}(\lambda)=\left\{M K_{\lambda}^{-1}\left(\frac{K_{\lambda}\left(z_{0}\right)}{M}\right):-1 \leqslant \lambda \leqslant 1\right\}
$$

where

$$
K_{\lambda}(z)=\frac{z}{1-2 \lambda z+z^{2}}, \quad-1 \leqslant \lambda \leqslant 1
$$

As for $\gamma_{2}$, it is parameterized as follows. Rewrite the Loewner ODE as a system of ODE for the two real phase coordinates of $x=\left(x_{1}, x_{2}\right), x_{1}(t)=\left|w\left(z_{0}, t\right)\right|$ 
and $x_{2}(t)=\arg w\left(z_{0}, t\right)$ with the main branch of the argument, $x_{1}(0)=\left|z_{0}\right|$ and $x_{2}(0)=\arg z_{0}$. Construct a Hamilton function $H(x, \Psi, u)$, where the vector $\Psi=\left(\Psi_{1}, \Psi_{2}\right)$ obeys the adjoint system of differential equations

$$
\frac{d \Psi_{1}}{d t}=-\frac{\partial H}{\partial x_{1}}, \quad \frac{d \Psi_{2}}{d t}=-\frac{\partial H}{\partial x_{2}},
$$

and $u=u^{*}(x, \Psi)$ is a maximum point of $H(x, \Psi, u)$ in $u$ for $u \in[-2,2]$. Next, solve the Cauchy problem for the system of four ODE for the phase and adjoint variables with the initial data for $\Psi$ such that $\Psi_{1}(0)$ is equal either $(-1)$ or 1 , and $\Psi_{2}(0)$ is a free parameter $\lambda$ parameterizing $\gamma_{2}(\lambda), \lambda \in E$. The set $E$ is determined by the condition that, for $\lambda \in E$, a unique maximum point $u_{0}=u^{*}(x(0), \Psi(0))$ of $H(x(0), \Psi(0), u)$ in $u$ belongs to $(-2,2)$. Then,

$$
\gamma_{2}(\lambda)=w\left(z_{0}, \log M, \lambda\right)
$$

and $w\left(z_{0}, t, \lambda\right)$ solves the Loewner ODE with $u(t)=u^{*}(x(t), \Psi(t))$ and $\lambda \in E$.

It is interesting to add that the adjoining vectors $\Psi(\log M)$ corresponding to the parameter $\lambda$ are normal vectors for $\gamma_{2}$ at inner points $\gamma_{2}(\lambda), \lambda \in E$.

\section{VALUE REGIONS FOR FUNCTIONS WITH FIXED BOUNDARY POINTS}

Since the seminal paper by Cowen and Pommerenke [36], the study of holomorphic functions with finite angular derivatives at prescribed points has been an active field of research in complex analysis.

Given a holomorphic function $f$ in $\mathbb{D}$ and a point $\sigma \in \partial \mathbb{D}$ such that there exists a finite angular limit $f(\sigma)=\angle \lim _{z \rightarrow \sigma} f(z)$, the angular derivative at $\sigma$ is

$$
f^{\prime}(\sigma)=\angle \lim _{z \rightarrow \sigma} \frac{f(z)-f(\sigma)}{z-\sigma} .
$$

On the one hand, for univalent functions $f$, existence of the angular derivative $f^{\prime}(\sigma)$ different from 0 and $\infty$ is closely related to the geometry of $f(\mathbb{D})$ near $f(\sigma)$. Moreover, if there exists $f^{\prime}(\sigma) \neq 0, \infty$, then the behavior of $f$ at the boundary point $\sigma$ resembles conformality.

On the other hand, for the dynamics of a holomorphic self-map $f: \mathbb{D} \rightarrow \mathbb{D}$, a crucial role is played by the points $\sigma \in \partial \mathbb{D}$ for which $f(\sigma)=\sigma$ and the angular derivative $f^{\prime}(\sigma)$ is finite, see e.g. [37].

Such points $\sigma$ are called boundary regular fixed points. In particular, a classical result due to Wolff and Denjoy asserts that if $f \in \operatorname{Hol}(\mathbb{D}, \mathbb{D})$ has no fixed points in $\mathbb{D}$, then it possesses the so-called boundary Denjoy - Wolff point, i.e., a unique boundary regular fixed point $\tau$ such that $f^{\prime}(\tau) \leqslant 1$.

Consider univalent self-maps $f: \mathbb{D} \rightarrow \mathbb{D}$ with a given boundary regular fixed point $\sigma \in \partial \mathbb{D}$ and the Denjoy - Wolff point $\tau \in \partial \mathbb{D} \backslash\{\sigma\}$. Using automorphisms of $\mathbb{D}$, we may suppose that $\tau=1$ and $\sigma=-1$ and look for a sharp value region of $f \mapsto f\left(z_{0}\right)$ for all such self-maps of $\mathbb{D}$ with $f^{\prime}(-1)$ fixed. Define the strip

$$
\mathbb{S}=\left\{\zeta:-\frac{\pi}{2}<\operatorname{Im} \zeta<\frac{\pi}{2}\right\}
$$

Let

$$
\zeta_{0}=x_{1}^{0}+i x_{2}^{0}:=\ell\left(z_{0}\right)
$$


where

$$
\ell: \mathbb{D} \rightarrow \mathbb{S}, z \mapsto \log \frac{1+z}{1-z},
$$

is a conformal map of $\mathbb{D}$ onto $\mathbb{S}$. For $T>0$, define

$$
\begin{gathered}
a_{ \pm}(T):=e^{-T / 2} \sin x_{2}^{0} \pm\left(1-e^{-T / 2}\right), \quad R(a, T):=\log \frac{1-a}{\left.1-a_{+}(T)\right)} \log \frac{1+a}{1+a_{-}(T)} \\
V\left(\zeta_{0}, T\right):=\left\{x_{1}+i x_{2} \in \mathbb{S}: a_{-}(T) \leqslant \sin x_{2} \leqslant a_{+}(T)\right. \\
\left.\left|x_{1}-x_{1}^{0}-T / 2\right| \leqslant \sqrt{R\left(\sin x_{2}, T\right)}\right\} .
\end{gathered}
$$

The following theorem gives a description of the value region $\left\{f\left(z_{0}\right)\right\}$ over the class of univalent self-maps $f: \mathbb{D} \rightarrow \mathbb{D}$ with two fixed boundary points \pm 1 .

Theorem 13 ([38]). Let $f \in \operatorname{Hol}(\mathbb{D}, \mathbb{D}) \backslash\left\{i d_{\mathbb{D}}\right\}$ and $T>0$. Suppose that

(i) $f$ is univalent in $\mathbb{D}$;

(ii) the Denjoy - Wolff point of $f$ is $\tau=1$;

(iii) $\sigma=-1$ is a boundary regular fixed point of $f$ and $f^{\prime}(-1)=e^{T}$.

Then

$$
f\left(z_{0}\right) \in \mathscr{V}\left(z_{0}, T\right):=\ell^{-1}\left(V\left(\ell\left(z_{0}\right), T\right)\right) \backslash\left\{z_{0}\right\} \text { for any } z_{0} \in \mathbb{D} .
$$

This result is sharp, i.e., for any $w_{0} \in \mathscr{V}\left(z_{0}, T\right)$ there exists $f \in \operatorname{Hol}(\mathbb{D}, \mathbb{D}) \backslash\left\{i d_{\mathbb{D}}\right\}$ satisfying (i)-(iii) and such that $f\left(z_{0}\right)=w_{0}$.

In the same paper, the authors also characterize functions $f$ corresponding to boundary points of $\mathscr{V}\left(z_{0}, T\right)$ in the last theorem. Let $K$ be the Koebe function

$$
K(z)=\frac{z}{(1-z)^{2}}
$$

Define the functions $p_{\alpha}$ as

$$
p_{\alpha}(z)=K^{-1}(\alpha K(z)), \quad \alpha \in(0,1),
$$

which map $\mathbb{D}$ onto $\mathbb{D} \backslash[-1,-r], r=r(\alpha) \in(0,1)$.

Since the Koebe function $K$ and its rotations are known to be extremal in many extremal problems and $p_{\alpha}$ plays the role of the Koebe function in the class of univalent functions $f: \mathbb{D} \rightarrow \mathbb{D}$, it is natural to expect that functions of the form $f=h_{1} \circ p_{\alpha} \circ h_{2}$, $h_{1}, h_{2} \in \operatorname{Aut}(\mathbb{D})$, would be extremal in the value region problem. The following theorem confirms these expectations.

Theorem 14 ([38]). For any $w_{0} \in \partial \mathscr{V}\left(z_{0}, T\right) \backslash\left\{z_{0}\right\}$, there exists a unique $f=$ $=f_{w_{0}}$ satisfying the conditions $(i)$-(iii) in the value region problem for univalent self-maps $f: \mathbb{D} \rightarrow \mathbb{D}$ with two boundary fixed points \pm 1 and such that $f_{w_{0}}\left(z_{0}\right)=w_{0}$. If $w_{0}=\ell^{-1}\left(\zeta_{0}+T\right)$, then $f_{w_{0}}$ is a hyperbolic automorphism of $\mathbb{D}$, namely, $f_{w_{0}}(z)=\ell^{-1}(\ell(z)+T)$. Otherwise, $f_{w_{0}}$ is a conformal mapping of $\mathbb{D}$ onto $\mathbb{D}$ minus a slit along an analytic Jordan arc $\gamma$ orthogonal to $\partial \mathbb{D}$, with $f_{w_{0}}^{\prime}(1)=1$. Moreover, $f_{w_{0}}=h_{1} \circ p_{\alpha} \circ h_{2}$ for some $h_{1}, h_{2} \in \operatorname{Aut}(\mathbb{D})$ and $\alpha \in(0,1)$ if and only if

$$
w_{0}=\ell^{-1}\left(x_{1}^{0}+\frac{T}{2}+i \arcsin a_{ \pm}(T)\right) \text {. }
$$


Note that $z_{0}$ is a boundary point of the value region $\mathscr{V}\left(z_{0}, T\right)$, but does not belong to $\mathscr{V}\left(z_{0}, T\right)$. However, $z_{0}$ would be included, and this would be the only modification of $\mathscr{V}\left(z_{0}, T\right)$, if the equality $f^{\prime}(-1)=e^{T}$ in the value region problem is replaced by the inequality $f^{\prime}(-1) \leqslant e^{T}$ and the requirement $f \neq \mathrm{id} \mathrm{d}_{\mathbb{D}}$ is removed and we assume as a convention that $\mathrm{id}_{\mathbb{D}}$ satisfies (ii). Under the theorem conditions modified in this way, $f\left(z_{0}\right)=z_{0}$ if and only if $f=\mathrm{id}_{\mathbb{D}}$.

If $f \in \operatorname{Hol}(\mathbb{D}, \mathbb{D})$ has boundary regular fixed points at \pm 1 , then replacing $f$ by $h \circ f$, where $h$ is a suitable hyperbolic automorphism with the same boundary fixed points, we may suppose that $\tau=1$ is the Denjoy - Wolff point. Hence, we deduce a sharp estimate for $f^{\prime}(-1) f^{\prime}(1)$, which was obtained earlier in [39] with the help of the extremal length method.

Corollary 1 ([38]). Let $z_{0} \in \mathbb{D}$ and let $f \in \operatorname{Hol}(\mathbb{D}, \mathbb{D})$ be a univalent function with boundary regular fixed points at 1 and (-1). Then

$$
\sqrt{f^{\prime}(-1) f^{\prime}(1)} \geqslant \max \left\{\frac{1+\sin \operatorname{Im} \ell\left(z_{0}\right)}{1+\sin \operatorname{Im} \ell\left(f\left(z_{0}\right)\right)}, \frac{1-\sin \operatorname{Im} \ell\left(z_{0}\right)}{1-\sin \operatorname{Im} \ell\left(f\left(z_{0}\right)\right)}\right\} .
$$

This inequality is sharp. The equality can occur only for hyperbolic automorphisms and functions $f$ of the form $f=h_{1} \circ p_{\alpha} \circ h_{2}, h_{1}, h_{2} \in \operatorname{Aut}(\mathbb{D}), \alpha \in(01)$.

Value region problems over classes $\mathscr{H}, \mathscr{H}(T), S, S(M), S_{R}, S_{R}(M)$ and others required the classic Loewner parametric representations by the chordal and radial differential equations and their modifications and generalizations. However, a parametric theory for classes of univalent self-maps with fixed boundary points was created in the last decades, mainly in papers due to Goryainov and his colleagues. Solutions to the value region problems for mappings with boundary fixed points are based on a new version of Loewner's parametric method, which was discovered recently by Goryainov and Kudryavtseva [40].

Theorem 15 ([40]). Let $\mathfrak{B}[q ; a]$ denote the class of holomorphic univalent self-maps $f: \mathbb{D} \rightarrow \mathbb{D}$ with a Denjoy - Wolff point $q$ and a boundary regular fixed point a and let $\left\{f^{t}\right\}_{t \geqslant 0}$ be a one-parametric semigroup $t \mapsto f^{t}$ which is a solution to the differential equation

$$
\frac{\partial f^{t}(z)}{\partial t}=v\left(f^{t}(z)\right),\left.\quad f^{t}(z)\right|_{t=0}=z .
$$

Then $\left\{f^{t}(z)\right\}_{t \geqslant 0} \subset \mathfrak{B}[q ; a]$ if and only if

$$
v(z)=\alpha(q-z)(1-\bar{q} z)(1-\bar{a} z) h(\bar{a} z),
$$

where $\alpha \geqslant 0$ and

$$
h(z)=\int_{\partial \mathbb{D}} \frac{1-æ}{1-æ z} d \mu(æ)
$$

with a probability measure $\mu$ on $\partial \mathbb{D}$.

Goryainov [41] obtained also the sharp value region $\left\{f^{\prime}(0)\right\}$ in the class of all univalent $f \in \operatorname{Hol}(\mathbb{D}, \mathbb{D}), f(0)=0$, having a boundary regular fixed point $\sigma=1$ with a given value of $f^{\prime}(1)$.

In [42], Goryainov proved that the value region $\mathscr{D}(0, T)$ of $f \mapsto f(0)$ over all selfmaps $f$ satisfying the conditions of the value region problem over self-maps $\mathbb{D} \rightarrow \mathbb{D}$ 
having two fixed boundary points without the univalence requirement is the closed disk whose diameter is the segment $\left[0, \ell^{-1}(T)\right]$, with the boundary point $z_{0}=0$ excluded. Analyzing the functions bringing the boundary points of $\mathscr{D}(0, T)$, one can conclude that $\partial \mathscr{D}(0, T) \cap \partial \mathscr{V}(0, T)=\left\{0, \ell^{-1}(T)\right\}$.

\section{COEFFICIENT VALUE REGIONS}

For $z_{0}=0$, a value region $\left\{f\left(z_{0}\right), f^{\prime}\left(z_{0}\right), \ldots, f^{(n)}\left(z_{0}\right)\right\}$ over a class of functions $f(z)=z+\sum_{n=2}^{\infty} a_{n} z^{n}, z \in \mathbb{D}$, is reduced to the coefficient value region

$$
\left\{a_{2}, \ldots, a_{n}\right\}=\left\{\frac{f^{\prime \prime}(0)}{2 !}, \ldots, \frac{f^{(n)}(0)}{n !}\right\}, \quad n \geqslant 2 .
$$

Denote

$$
V_{n}=\left\{\left(a_{2}, \ldots, a_{n}\right): f \in S\right\}, \quad V_{m n}=\left\{\left(a_{n}, a_{m}\right): f \in S\right\} \quad n, m \geqslant 2,
$$

and

$$
\mathscr{M}_{n}=\left\{\left(a_{2}, \ldots, a_{n}\right): f \in \tilde{S}\right\}, \quad n \geqslant 2,
$$

where $\tilde{S}$ stands for the class of functions $f \in S$ which are $C^{\infty}$-smooth on $\partial \mathbb{D}$. A good collection of qualitative results is contained in [9,43]. Namely:

(i) $V_{n}$ is homeomorphic to a $(2 n-2)$-dimensional closed ball, and its boundary $\partial V_{n}$ is homeomorphic to a $(2 n-3)$-dimensional sphere;

(ii) every point $x \in \partial V_{n}$ corresponds to exactly one function $f \in S$;

(iii) with the exception for a set of smaller dimension, at every point $x \in \partial V_{n}$ there exists a normal vector satisfying the Lipschitz condition;

(iv) there exists a connected open set $X_{1} \subset \partial V_{n}$, such that $\partial V_{n}$ is an analytic hypersurface at every point of $X_{1}$. The points of $\partial V_{n}$ corresponding to the functions that give the extremum to a linear functional belong to the closure of $X_{1}$.

On an $n$-dimensional manifold $\mathscr{M}_{n}$, a sub-Riemannian structure is a smoothly varying distribution $\mathscr{D}$ of $k$ planes together with a scalar product. The distribution $\mathscr{D}$ is a linear sub-bundle of a tangent bundle $T \mathscr{M}_{n}$ of $\mathscr{M}_{n}$. If $k<n$, then the Hausdorff dimension of $\mathscr{M}_{n}$ is larger than $n$. Suppose that a system of vector fields $X_{1}, \ldots, X_{k}$ form an orthonormal basis of $\mathscr{D}$ with respect to scalar product $\langle\cdot, \cdot\rangle$. The pair $(\mathscr{D},\langle\cdot, \cdot\rangle)$ is a sub-Riemannian metric on $\mathscr{M}_{n}$. Let all vector fields $X_{1}, \ldots, X_{k}$ together with their commutators form the total tangent space $T \mathscr{M}_{n}$. If the number of these commutators is independent of the point of $\mathscr{M}_{n}$, then it is said that $X_{1}, \ldots, X_{k}$ satisfy the bracket generating condition.

Theorem 16 ([44]). Let $\mathscr{M}_{n}$ be the coefficient value region for $\tilde{S}$ and let $L_{1}, \ldots, L_{n}$ be the vector fields defined by

$$
L_{j}=\partial_{j}+\sum_{k=1}^{n-j}(k+1) a_{k} \partial_{j+k}, \quad \partial_{j}=\frac{\partial}{\partial a_{j}} .
$$

Then the system $\left(L_{1}, L_{2}\right)$ satisfies the bracket generating condition and the distribution is $\mathscr{D}=\operatorname{span}\left(L_{1}, L_{2}\right)$.

The Hausdorff complex dimension of the sub-Riemannian manifold $\mathscr{M}_{n}$ is equal to $\left(\frac{n}{2}+1\right)^{2}-\frac{9}{2}$ for odd $n$ and is equal to $\left(\frac{n}{2}+1\right)^{2}-2$ for even $n$. 
Call attention to the theory of integrable systems in connection with the Loewner equation. Let $\lambda(z, \mathbf{t})$ be a meromorphic function in $z$ that depends on an infinite family of generated times $\mathbf{t}=\left(t_{0}, t_{1} \ldots, t_{n}, \ldots\right), t_{0}=x$, with the expansion

$$
\lambda(z, \mathbf{t})=z+\sum_{n=0}^{\infty} \frac{A^{n}(\mathbf{t})}{z^{n+1}}
$$

about infinity. The dispersionless Kadomtsev - Petviashvili (dKP) hierarchy is an infinite set

$$
\frac{\partial \lambda}{\partial t_{n}}=\left\{\mathscr{L}_{n+1}, \lambda\right\}, \quad n=0,1,2, \ldots,
$$

where $\{\cdot, \cdot\}$ is the Poisson bracket

$$
\begin{gathered}
\{F, G\}=\frac{\partial F}{\partial z} \frac{\partial G}{\partial x}-\frac{\partial F}{\partial x} \frac{\partial G}{\partial z} \\
\mathscr{L}_{n}=\frac{1}{n}\left(\lambda^{n}\right)_{\geqslant 0}, \quad n=1,2, \ldots,
\end{gathered}
$$

denotes the polynomial part of $\lambda^{n}$.

A finite-dimensional reduction suggests that $\lambda$ depends on $\mathbf{t}$ via a finite number of functions, $\lambda(z, \mathbf{t})=\lambda\left(z, u_{1}(\mathbf{t}), \ldots, u_{N}(z, \mathbf{t})\right)$. Takebe, Teo and Zabrodin [45] showed that the chordal Loewner PDE serves as the consistency condition for the one-variable reduction of the dKP. A function $\lambda$ depends on $\mathbf{t}$ via one function $s(\mathbf{t})$ and

$$
\frac{\partial \lambda}{\partial s}=-\frac{\kappa}{z-\xi} \frac{\partial \lambda}{\partial z}
$$

which is the Loewner PDE, where $\kappa$ is the derivative in $s$ of the coefficient at $1 / z$ in the Laurent expansion of $\lambda$.

In [46], the authors showed that the chordal Loewner evolution has an infinite number of conservation laws, i.e., moments, and that the Loewner PDE is exactly the Vlasov equation under an appropriate change of variables, that the Loewner ODE implies the hydrodynamic-type conservation equation. Starting with the Loewner evolution, the authors obtain integrable chains by splitting time. This approach demonstrates the universality of the Loewner equation as an attraction point for several integrable chains.

Mention that, for the function $f(z, t(x, s))=f(z, x, s)$, the Vlasov equation

$$
z \frac{\partial f}{\partial x}+\frac{\partial f}{\partial s}-\frac{\partial A^{0}}{\partial x} \frac{\partial f}{\partial z}=0
$$

describes the time evolution of the distribution function of a plasma consisting of charged particles with long-range interaction.

Going back to the coefficient value region $V_{n}$, recall the exclusive role of the Koebe function $K(z)=\sum_{n=1}^{\infty} n z^{n}$, especially in the connection with the famous Bieberbach conjecture stating that the inequality $\left|a_{n}\right| \leqslant n$ holds for all $f \in S$ with the equality sigh only for $K(z)$ and its rotations.

Studying the local version of the Bieberbach conjecture, Bombieri, the Fields medalist, was interested in metric and geometric properties of $V_{n}$ in a neighborhood the point 
$\left(a_{2}, \ldots, a_{n}\right)=(2, \ldots, n) \in \partial V_{n}$ corresponding to $K(z)$. Bombieri [47] conjectured that the numbers

$$
\sigma_{m n}:=\liminf _{S \ni f \rightarrow K} \frac{n-\operatorname{Re} a_{n}}{m-\operatorname{Re} a_{m}}, \quad m, n \geqslant 2,
$$

where $f \rightarrow K$ locally uniformly in $\mathbb{D}$, are equal to the numbers

$$
B_{m n}:=\min _{\theta \in[0,2 \pi]} \frac{n \sin \theta-\sin (n \theta)}{m \sin \theta-\sin (m \theta)}
$$

for all $m, n \geqslant 2$.

Bombieri's number $\sigma_{m n}$ determines a locally support hyperplane given by

$$
\operatorname{Re}\left(a_{n}-\sigma_{m n} a_{m}\right)=n-\sigma_{m n} m
$$

in a neighborhood of the point $(n, m) \in V_{m n}$ corresponding to $K$. It is proved in [48] that $\sigma_{m n}$ is a maximal number for which $K$ locally maximizes the linear functional,

$$
\sigma_{m n}=\sup \left\{\lambda \in \mathbb{R}: \operatorname{Re}\left(a_{n}-\lambda a_{m}\right) \text { is locally maximized on } S \text { by } K(z)\right\} \text {. }
$$

For $(m, n)=(3,2)$, this property has been proved by Greiner and Roth in [49]. Such interpretation can serve to define a number $\sigma_{m n}(M), M>1$, as a maximal number for which $\operatorname{Re}\left(a_{n}-\lambda a_{m}\right)$ is locally maximized on $S(M)$ by the Pick function $P_{M}(z)=M K^{-1}(K(z) / M)$. Values $\sigma_{23}(M)$ and $\sigma_{32}(M), M>1$, are evaluated in [50] and [51], respectively.

Theorem 17 ([50,51]). For $1<M \leqslant \infty$, we have

$$
\sigma_{23}(M)=-\frac{4}{M}
$$

and

$$
\sigma_{32}(M)= \begin{cases}\frac{M(e-1)}{4(M e-2 e+1)}, & e \leqslant M \leqslant \infty \\ \frac{M}{4(M-1)}, & 1<M \leqslant e .\end{cases}
$$

Bombieri's conjecture is true for the class $S_{R}$, see the proof due to Bshouty and Hengartner in [52]. The inequality $\sigma_{m n} \leqslant B_{m n}$ was proved in [48] for all $m, n \geqslant 2$. Hence, $\sigma_{m n}=0$ when $B_{m n}=0$.

Greiner and Roth [49] answered negatively to partial Bombieri's conjecture. They evaluated $\sigma_{32}$ showing that $\sigma_{32}<B_{32}$. Later, $\sigma_{42}, \sigma_{24}$ and $\sigma_{34}$ were evaluated in [53], each time disproving Bombieri's conjecture for these pairs of indices. Related versions of Bombieri's conjecture are discussed by Aharonov and Bshouty in [54]. Using the linear Loewner PDE, Leung [55] derived a variational formula in $S$ near the Koebe function $K$ and disproved Bombieri's conjecture for index pairs $(m, 2)$ with $m>2$ and for $(m, 3)$ with odd $m>3$. In particular, Leung's variation leads to a statement that Bombieri's conjecture fails for $(m, n)$ when

$$
B_{m n}=\frac{n^{3}-n}{m^{3}-m}
$$


Efraimidis [56] and Efraimidis and Pastor [57] selected a lot of pairs $(m, n)$ for which $B_{m n}$ are given by the latter expression. Namely, they showed that $B_{m n}$ satisfies (5), i.e., Bombieri's conjecture fails, for the following integers $m$ and $n, m>n \geqslant 2$,

(a) both $m$ and $n$ are odd, or

(b) both $m$ and $n$ are even, or

(c) $m$ is even, $n$ is odd, $0.5 \leqslant n / m \leqslant 0.8194$ and $m \geqslant 81$.

Necessary criteria for the Bombieri conjecture are derived in [58] in terms of inequalities for solutions to systems of differential equations in variations for the Loewner ODE.

We see that Bombieri's conjecture is attacked by different methods: Schiffer's differential equation for support points of $S[48,49]$, variational method in the class $S$ [52, 55-57], Lebedev-Milin inequalities based on the Grunsky approach [54], the Loewner theory and optimal control methods and the Pontryagin maximum principle on solutions to the Loewner ODE [47,50,51,53,58]. Roth [27] gave a statement of Teichmüller's coefficient theorem entirely in terms of the Loewner equation. This allowed him to compare Schiffer's differential equation and Pontryagin's maximum principle. Roth concluded that two necessary conditions for $f \in S$ being extremal for a coefficient functional over $S$ provided by Schiffer's theorem and by Pontryagin's maximum principle are in fact equivalent. Roth claims that the Schiffer differential equation is analogous to the Euler equation in the classical calculus of variations and notices that the implication from Schiffer's equation to Pontryagin's maximum principle traces back to early periods in the geometric function theory.

In author's papers cited in this survey, we are focused on the optimal control theory methods applied to Bombieri's problem. We introduce the Loewner ODE and generate a control system for the coefficients of functions $f(z, t) \in S$ from a Loewner chain, $z \in \mathbb{D}$, $t \geqslant 0$. Following an optimization scheme, we deduce an adjoint control system of ODE and build a pseudo Hamilton function $H$ depending on a phase coefficient vector $a(t)$ and an adjoint vector $\bar{\Psi}(t)$, on a driving function $u$ and also on time $t$. The Pontryagin maximum principle is a necessary condition for the reachable set in a neighborhood of the extremal control $u=\pi$ corresponding to the Koebe function $K$. As far as $K$ is extremal in the Bombieri problem, the control $u=\pi$ has to satisfy the Pontryagin maximum principle, i.e., it maximizes $H$ on a whole trajectory $(a(t), \bar{\Psi}(t))$.

Acknowledgements: This work was supported by the Russian Science Foundation (project No. 17-11-01229).

\section{References}

1. Goluzin G. M. Geometric theory of functions of a complex variable. New York, Amer. Math. Soc., 1969. 684 p. (Russ. ed.: Moscow, Nauka, 1966. 628 p.).

2. Aleksandrov I. A. Parametrichrskie prodolzheniya v teorii odnolistnykh funktsij [Parametric continuations in the theory of univalent functions]. Moscow, Nauka, 1976. 344 p. (in Russian).

3. Duren P. L. Univalent functions. New York, Springer Verlag, 1983. 382 p.

4. Pommerenke Ch. Univalent functions. Göttingen, Vandenhoeck \& Ruprecht, 1975. 376 p.

5. Rogosinski W. Zum Schwarzen Lemma. Jahresber. Deutsche Math-Verein., 1934, vol. 44, pp. 258-261.

6. Grunsky H. Neue Abschätzungen zur konformen Abbildung ein- und mehrfach zusammenhängender Bereiche. Schr. Math. Inst. u Inst. Angew. Math. Univ. Berlin, 1932, vol. 1, pp. 95-140. 
7. Popov V. I. Value domain of a system of functionals on the class S. Trudy Tomskogo universiteta. Voprosy geometricheskoj teorii funktsij [Proceedings of Tomsk University. Issues of the geometric function theory], 1965, vol. 182, iss. 3, pp. 106-132 (in Russian).

8. Gutlyanskii V. Ya. Parametric representation of univalent functions. Soviet Math. Dokl., 1970, vol. 11, no. 5, pp. 1273-1276.

9. Schaeffer A. C., Spencer D. C. Coefficient regions for schlicht functions. Coll. Publ., vol. 35. New York, Amer. Math. Soc., 1950. 325 p.

10. Loewner K. Untersuchungen über schlichte konforme Abbildungen des Einheitskreises. I. Math. Ann., 1923, vol. 89, no. 1-2, pp. 103-121.

11. Kufarev P. P. On one-parameter families of analytic functions. Rec. Math. [Mat. Sbornik] N.S., 1943, vol. 13 (55), no. 1, pp. 87-118 (in Russian).

12. Pommerenke Ch. Über die Subordination analytischer Funktionen. J. Reine Angew. Math., 1965, vol. 218, pp. 159-173.

13. Kufarev P. P. A remark on integrals of the Loewner equation. Doklady Akad. Nauk SSSR, 1947, vol. 57, no. 7, pp. 655-656 (in Russian).

14. Goryainov V. V., Gutlyanskii V. Ya. On extremal problems in the class $S_{M}$. In: Matematicheskii sbornik. Kiev, Naukova dumka, 1976, pp. 242-246 (in Russian).

15. Roth O., Schleissinger S. Rogosinski's lemma for univalent functions, hyperbolic Archimedean spirals and the Loewner equation. Bull. London Math. Soc., 2014, vol. 46, no. 5, pp. 1099-1109. DOI: https://doi.org/10.1112/blms/bdu054

16. Prokhorov D., Samsonova K. Value range of solutions to the chordal Loewner equation. J. Math. Anal Appl., 2015, vol. 428, no. 2, pp. 910-919. DOI: https://doi.org/10.1016/j.jmaa.2015.03.065

17. Zherdev A. Value range of solutions to the chordal Loewner equation with restriction on the driving function. Probl. Anal. Issues Anal, 2019, vol. 8(26), no. 2, pp. 92-104. DOI: https://doi.org/10.15393/j3.art.2019.6270

18. Koch J. D. Value regions for schlicht functions. Dissertationsschrift zur Erlangung des naturwissenschaftlichen Doktorgrades der Julius-Maximilians-Universität Würzburg. Würzburg, 2016. 93 p.

19. Goodman G. S. Univalent functions and optimal control. Ph.D. Thesis, Stanford University, 1967. ProQuest LLC, Ann Arbor, MI.

20. Friedland S., Schiffer M. Global results in control theory with applications to univalent functions. Bull. Amer. Math. Soc., 1976, vol. 82, no. 6, pp. 913-915.

21. Friedland S., Schiffer M. On coefficient regions of univalent functions. J. Anal. Math., 1977, vol. 31, pp. 125-168.

22. Roth O. Control Theory in $\mathscr{H}(\mathbb{D})$. Dissertation zur Erlangung des naturwissenschaftlichen Doktorgrades der Bayerischen Julius-Maximilians- Universität Würzburg. Würzburg, 1998. 178 p.

23. Prokhorov D. Sets of values of systems of functionals in classes of univalent functions. Mat. Sb., 1990, vol. 181, no. 12, pp. 1659-1677; Math. USSR-Sb., 1992, vol. 71, no. 2, pp. 499-516.

24. Prokhorov D. Reachable set methods in extremal problems for univalent functions. Saratov, Saratov Univ. Press, 1993. 228 p.

25. Schiffer M. Sur l'équation différentielle de M. Löwner. C. R. Acad. Sci. Paris, 1945, vol. 221, pp. 369-371.

26. Prokhorov D., Vasil'ev A. Univalent functions and integrable systems. Commun. Math. Phys., 2006, vol. 262, no. 2, pp. 393-410. DOI: https://doi.org/10.1007/s00220-0051499-y

27. Roth O. Is there a Teichmüller principle in higher dimension? Geometric function theory in higher dimension. F. Bracci (ed.). Cham, Springer INdAM Series 26, 2017, pp. 87-105. DOI: https://doi.org/10.1007/978-3-319-73126-1_7 
28. Popov V. I. L. S. Pontryagin's maximum principle in the theory of univalent functions. Soviet Math. Dokl., 1969, vol. 10, pp. 1161-1164.

29. Prokhorov D. The method of optimal control in an extremal problem on a class of univalent functions. Soviet Math. Dokl., 1984, vol. 29, pp. 301-303.

30. Prokhorov D. Bounded univalent functions. Handbook of complex analysis: Geometric function theory, vol. 1. Amsterdam, North Holland, 2002, pp. 207-228.

31. Koch J., Schleissinger S. Value ranges of univalent self-mappings of the unit disc. J. Math. Anal. Appl., 2016, vol. 433, no. 2, pp. 1772-1789. DOI: https://doi.org/10.1016/j.jmaa.2015.08.068

32. Koch J., Schleissinger S. Three value ranges for symmetric self-mappings of the unit disk. Proc. Amer. Math. Soc., 2017, vol. 145, no. 4, pp. 1747-1761. DOI: https://doi.org/10.1090/proc/13350

33. Fedorov S. I. The moduli of certain families of curves and the range of $\left\{f\left(\zeta_{0}\right)\right\}$ in the class of univalent functions with real coefficients. J. Soviet Math., 1987, vol. 36, pp. 282-291.

34. Jenkins J.A. On univalent functions with real coefficients. Ann. Math., 1960, vol. 71, pp. $1-15$.

35. Prokhorov D., Samsonova K. A description method in the value region problem. Complex Anal. Oper. Theory, 2017, vol. 11, no. 7, pp. 1613-1622. DOI: https://doi.org/10.1007/s11785-016-0551-6

36. Cowen C. C., Pommerenke Ch., Inequalities for the angular derivative of an analytic function in the unit disk. J. London Math. Soc., 1982, vol. 26, no. 2, pp. 271-289. DOI: https://doi.org/10.1112/jlms/s2-26.2.271

37. Goryainov V. V. Fractional iterates of functions that are analytic in the unit disk with given fixed points. Math. USSR-Sb., 1993, vol. 74, no. 1, pp. 29-46. DOI: https://doi.org/10.1070/SM1993v074n01ABEH003332

38. Gumenyuk P., Prokhorov D. Value regions of univalent self-maps with two boundary fixed points. Ann. Acad. Sci. Fenn. Math., 2018, vol. 43, no. 1, pp. 451-462. DOI: https://doi.org/10.5186/aasfm.2018.4321

39. Frolova A., Levenshtein M., Shoikhet D., Vasil'ev A. Boundary distortion estimates for holomorphic maps. Complex Anal. Oper. Theory, 2014, vol. 8, no. 5, pp. 1129-1149. DOI: https://doi.org/10.1007/s11785-013-0345-z

40. Goryainov V. V., Kudryavtseva O. S. One-parameter semigroups of analytic functions, fixed points and the Koenigs function. Sb. Math., 2011, vol. 202, no. 7-8, pp. 971-1000. DOI: https://doi.org/10.1070/SM2011v202n07ABEH004173

41. Goryainov V. V. Evolution families of conformal mappings with fixed points and the Loewner-Kufarev equation. Sb. Math., 2015, vol. 206, no. 1-2, pp. 33-60. DOI: https://doi.org/10.1070/SM2015v206n01ABEH004445

42. Goryainov V. V. Holomorphic mappings of the unit disc into itself with two fixed points. Sb. Math., 2017, vol. 208, no. 3, pp. 360-376. DOI: https://doi.org/10.1070/SM8802

43. Babenko K. I. The theory of extremal problems for univalent functions of class $S$. Providence, RI, Amer. Math. Soc., 1975. 320 p.

44. Markina I., Prokhorov D., Vasil'ev A. Sub-Riemannian geometry of the coefficients of univalent functions. J. Funct. Anal., 2007, vol. 245, no. 2, pp. 475-492. DOI: https://doi.org/10.1016/j.jfa.2006.09.013

45. Takebe T., Teo L.-P., Zabrodin A. Löwner equation and dispersionless hierarchies. J. Phys. A: Math. Theor., 2006, vol. 39, no. 37, pp. 11479-11501. DOI:10.1088/03054470/39/37/010

46. Pavlov M. V., Prokhorov D. V., Vasil'ev A. Yu., Zakharov A. M. Löwner evolution and finite dimensional reductions of integrable systems. Theor. Math. Phys., 2014, vol. 181, no. 1, pp. 1263-1278. DOI: https://doi.org/10.1007/s11232-014-0211-9 
47. Bombieri E. On the local maximum property of the Koebe function. Invent. Math., 1967, vol. 4, pp. 26-67.

48. Prokhorov D., Roth O. On the local extremum property of the Koebe function. Math. Proc. Cambr. Phil. Soc., 2004, vol. 136, no. 2, pp. 301-312. DOI: https://doi.org/10.1017/S030500410300714X

49. Greiner R., Roth O. On support points of univalent functions and a disproof of a conjecture of Bombieri. Proc. Amer. Math. Soc., 2001, vol. 129, no. 12, pp. 3657-3664. DOI: https://doi.org/10.1090/S0002-9939-01-05994-9

50. Gordienko V., Prokhorov D. Analogy of Bombieri's number for bounded univalent functions. Lobacherskii J. Math., 2017, vol. 38, no. 3, pp. 429-434. DOI: https://doi.org/10.1134/S1995080217030118

51. Gordienko V., Prokhorov D. The Bombieri Problem for Bounded Univalent Functions. Math. Notes, 2019, vol. 105, no. 3-4, pp. 442-450. DOI: https://doi.org/10.1134/S0001434619030040

52. Bshouty D., Hengartner W. A variation of the Koebe mapping in a dense subclass of $S$. Canad J. Math., 1987, vol. 39, no. 1, pp. 54-73. DOI: https://doi.org/10.4153/CJM-1987004-8

53. Prokhorov D., Vasil'ev A. Optimal control in Bombieri's and Tammi's conjectures. Georgian Math. J., 2005, vol. 12, no. 4, pp. 743-761. DOI: https://doi.org/10.1515/GMJ.2005.743

54. Aharonov D., Bshouty D. A problem of Bombieri on univalent functions. Comput. Methods Funct. Theory, 2016, vol. 16, iss. 4, pp. 677-688. DOI: https://doi.org/10.1007/s40315016-0165-Z

55. Leung Y.-J. On the Bombieri numbers for the class S. J. Anal., 2016, vol. 24, no. 2, pp. 229-250. DOI: https://doi.org/10.1007/s41478-016-0017-2

56. Efraimidis I. On the failure of Bombieri's conjecture for univalent functions. Comput. Methods Funct. Theory, 2018, vol. 18, iss. 3, pp. 427-438. DOI: https://doi.org/10.1007/s40315-017-0222-2

57. Efraimidis I., Pastor C. Some more counterexamples for Bombieri's conjecture on univalent functions. https://arxiv.org:1710.10426v1[math.CV] (28 Oct. 2017).

58. Prokhorov D. Necessary criteria for the Bombieri conjecture. Anal. Math. Phys., 2018, vol. 18, no. 4, pp. 679-690. DOI: https://doi.org/10.1007/s13324-018-0248-2

Cite this article as:

Prokhorov D. V. Value Regions in Classes of Conformal Mappings. Izv. Saratov Univ. (N. S.), Ser. Math. Mech. Inform., 2019, vol. 19, iss. 3, pp. 258-279. DOI: https://doi.org/10.18500/1816-9791-2019-19-3-258-279

\section{УДК 517.54}

\section{Области значений в классах конфрормных отображений}

\section{Д. В. Прохоров}

Прохоров Дмитрий Валентинович, доктор фризико-математических наук, заведующий кафредрой математического анализа, Саратовский национальный исследовательский государственный университет имени Н. Г. Чернышевского, Россия, 410012, г. Саратов, ул. Астраханская, д. 83; научный сотрудник, Петрозаводский государственный университет, Россия, Республика Карелия, 185910, г. Петрозаводск, просп. Ленина, д. 33, ProkhorovDV@info.sgu.ru 
Обзор преимущественно посвящен недавним результатам в решении задачи об областях значений в различных классах голоморфных однолистных функций, представимых решениями дифрференциальных уравнений Левнера как в радиальной, так и в хордовой версиях. Важно также представить классические и современные методы решения и сравнить их эфрфективность. Наиболее подробно затронуты методы оптимизации и, в частности, принцип максимума Понтрягина. Областью значений является множество $\left\{f\left(z_{0}\right)\right\}$ всех возможных значений функционала $f \mapsto f\left(z_{0}\right)$, где $z_{0}$ - это фриксированная точка из верхней полуплоскости в хордовом случае или в единичном круге в радиальном случае, а $f$ пробегает класс конформных отображений. Решения дифрференциальных уравнений Левнера образуют плотные подклассы рассматриваемых семейств фрункций. Области значений коэфрфициентов $\left\{\left(a_{2}, \ldots, a_{n}\right): f(z)=z+\sum_{n=2}^{\infty} a_{n} z^{n}\right\},|z|<1$, составляют часть поля исследований, тесно связанного с экстремальными задачами и с гипотезой Бомбиери о структуре области значений коэфрфрициентов на классе $S$ в окрестности точки $(2, \ldots, n)$, соответствующей фрункции Кебе.

Ключевые слова: область значений, уравнение Левнера, достижимое множество, граничная кривая.

Поступила в редакцию: 07.04.2018 / Принята: 12.05.2019 / Опубликована: 31.08.2019

Статья опубликована на условиях лицензии Creative Commons Attribution License (CC-BY 4.0)

Благодарности. Исследование выполнено при финансовой поддержке Российского научного фонда (проект № 17-11-01229).

\section{Образец для цитирования:}

Prokhorov D. V. Value Regions in Classes of Conformal Mappings [Прохоров Д. В. Области значений в классах конформных отображений] // Изв. Сарат. ун-та. Нов. сер. Сер. Математика. Механика. Информатика. 2019. Т. 19, вып. 3. С. 258-279. DOI: https://doi.org/10.18500/1816-9791-2019-19-3-258-279 